\title{
HEPATECTOMIA EM PACIENTES IDOSOS
}

\author{
Liver ressection in elderly patients
}

\author{
Gustavo Andreazza LAPORTE, Antônio Nocchi KALIL
}

\begin{abstract}
Trabalho realizado no Programa de PósGraduação de Ciências da Saúde da Universidade Federal de Ciências da Saúde de Porto Alegre e Serviço de Oncologia Cirúrgica do Hospital Santa Rita da Irmandade da Santa Casa de Misericórdia de Porto Alegre, Porto Alegre, RS, Brasil.
\end{abstract}

DESCRTIORES- Idoso. Hepatectomia Complicações pós-operatórias. Morbidade. Mortalidade.
RESUMO - Introdução - A expectativa de vida da população tem aumentado nos últimos anos, como também houve progressão da incidência de neoplasias primárias e secundárias do fígado. A medicina vem acompanhando esse processo, mas ainda há receios quanto ao uso de tratamentos cirúrgicos agressivos em pacientes idosos, especialmente no que tange à cirurgia do fígado. Objetivo - Analisar a influência da idade na morbimortalidade de pacientes submetidos à ressecção hepática. Métodos - Revisão bilbliográfica através dos sites do PubMed, Scielo e Bireme, com os descritores "elderly", "hepatectomy", "hepatic resection", "postoperative complications", "morbidity", "mortality". Foram selecionados os trabalhos que compararam os resultados de hepatectomia entre grupos de pacientes jovens e idosos. Conclusão - A idade não é fator predisponente à piora dos resultados na ressecção hepática.

\section{Correspondência:}

Gustavo Andreazza Laporte

E-mail: laportegustavo@gmail.com

Fonte de financiamento: não há

Conflito de interesses: não há

Recebido para publicação: 25/04/2012

Aceito para publicação: 27/11/2012

HEADINGS - Aged. Hepatectomy. Postoperative complications. Morbidity. Mortality.
ABSTRACT - Introduction - Life expectancy of the population has increased in recent years as a result of human development, leading to an increase in the incidence of primary and secondary neoplasms of the liver. Medicine has been following this process, but there is still fear of aggressive surgical treatment in elderly patients, especially concerning to liver surgery. Aim - To analyze the influence of age on the morbidity and mortality of patients undergoing liver resection. Method - Literature review of scientific articles available in PubMed, Scielo and Bireme database with "elderly", "hepatectomy," "hepatic resection," "postoperative complications", "morbidity", "mortality". It was selected studies that compared the results of hepatectomy between groups of young and elderly patients. Conclusion - Age is not a predisposing factor for the worsening of results in liver resection.

\section{INTRODUÇÃO}

expectativa de vida mundial aumentou muito nas últimas décadas,
acarretando envelhecimento populacional. Esse processo é
decorrente de diversos fatores como o aumento da taxa de fecundidade prevalente no passado em comparação com a atual, redução da mortalidade infantil, implementação de políticas governamentais de apoio à pessoa idosa, melhorias nas condições de trabalho, acesso aos serviços de saúde pública e alimentação de melhor qualidade. Também contribui para esse quadro a melhora das condições sociais e o incessante desenvolvimento dos avanços tecnológicos e do conhecimento médico?.

O Instituto Brasileiro de Geografia e Estatística demonstrou o aumento demográfico que alterou a pirâmide etária mundial e brasileira, levando à importantes mudanças sociais, políticas e sanitárias. A senescência, por si, resulta no aparecimento de doenças crônicas que podem afetar a qualidade e a funcionalidade na vida nos idosos. Por exemplo, eles são mais frequentemente candidatos à procedimentos cirúrgicos, seja na urgência ou eletivamente.

Com o envelhecimento da população, segundo o Instituto Nacional do Câncer, o Programa EUROCARE-3 e a International Society for Geriatric Oncology, a prevalência de câncer atinge proporções endêmicas, sendo que 
cerca de metade das neoplasias é diagnosticada em pacientes idosos ${ }^{12,21}$.

Dentro do contexto dos princípios do tratamento oncológico, a ressecção hepática é o tratamento de escolha para diversas doenças primárias e secundárias do fígado. A maioria dos estudos em idosos submetidos a esse procedimento reportou ressecção de tumores hepáticos primários e secundários, destacando-se o carcinoma hepatocelular e metástases do câncer colorretal $^{3}$. Contudo, observou-se nas últimas duas décadas que as hepatectomias tornaram-se seguras e realizadas também na população com idade mais avançada, implicando mudança de paradigma na abordagem destes pacientes ${ }^{15}$.

Este trabalho busca fornecer informações sobre a hepatectomia em pacientes idosos com o objetivo de auxiliar na decisão cirúrgica, valorizando a menor morbimortalidade e maior qualidade de vida desse grupo etário.

\section{MÉTODO}

Foi feita revisão bilbliográfica através dos sites do PubMed, Scielo e Bireme, com os descritores "elderly", "hepatectomy", "hepatic resection", "postoperative complications", "morbidity", "mortality". Foram selecionados os trabalhos que compararam os resultados de hepatectomia entre grupos de pacientes jovens e idosos.

\section{Tumores hepatobiliares}

Os tumores malignos hepatobiliares possuem sua incidência máxima entre a sexta e a oitava décadas de vida, com diagnóstico mais prevalente acima de 65 $\operatorname{anos}^{22}$.

A ressecção cirúrgica é o único tratamento potencial curativo para a maioria das neoplasias hepatobiliares primárias e secundárias, quando ressecáveis e limitadas. Além disso, o envelhecimento populacional, associado ao pico de incidência dos tumores malignos primários e secundários do fígado no segmento etário de 65 a 85 anos, resultou em aumento constante e dramático do número de pacientes idosos submetidos à ressecção hepática. Infelizmente, muitos médicos consideram que a idade avançada é contraindicação ao tratamento cirúrgico curativo tanto pelo alto risco, quanto por sobrevida limitada dos idosos. Muitas vezes, a operação não é oferecida em função de que os pacientes não são encaminhados a cirurgiões hepatobiliares e, assim, sofrem as consequências de tratamento inadequado ${ }^{17}$.

Um argumento utilizado de forma inapropriada para a definição de tratamento inadequado é o viés da idade, com a justificativa de que esses pacientes possuem expectativa de vida baixa em comparação aos mais jovens ${ }^{17}$. Entretanto, a expectativa de vida no Brasil, segundo dados do IBGE, em 2008, para uma pessoa de
60 anos de idade, é de 21,3 anos. Esse dado demográfico de nosso país, por si só pode justificar que os pacientes idosos com tumores malignos hepatobiliares devem ter tratamento adequado enquanto sua doença estiver limitada e suas comorbidades estiverem controladas.

No mundo, aproximadamente 427 mil pessoas morrem por carcinoma hepatocelular $(\mathrm{CHC})$, sendo a quarta causa de morte por câncer no mundo ${ }^{18}$. O pico de incidência, em torno da sexta década de vida, combinado com o envelhecimento populacional, resulta em aumento da população idosa com $\mathrm{CHC}$ considerada para ressecção hepática. Uma justificativa, já observada em modelos experimentais, é que, com o avançar da idade, há maior dano de DNA pelo estresse oxidativo e atuação de agentes carcinógenos, levando, assim, à alterações neoplásicas.

Em algumas séries, foi verificado que mais da metade de pacientes com $\mathrm{CHC}$ tem mais de 65 anos de idade e, destes, mais de $80 \%$ sofrem de cirrose. A sobrevida observada dos pacientes idosos é menor do que a dos pacientes jovens, geralmente pelo fato de que esses pacientes têm o seu diagnóstico mais tardio ou são tratados de forma inadequada. No entanto, quando se compara os mesmos estágios em pacientes ressecados, a sobrevida é semelhante entre os grupos, variando entre 18 e $76 \%$ em cinco anos ${ }^{17}$.

Dentre os preditores de pior sobrevida em idosos, assim como na população de pacientes mais jovens, figuram a cirrose e a classificação de Child $\mathrm{B} / \mathrm{C}^{22}$. Outra peculariedade observada foi que, em pacientes idosos, há maior taxa de infecção pelo vírus da hepatite $\mathrm{C}$ e baixa da hepatite $\mathrm{B}$. Também, há maior proporção de mulheres, sugerindo hepatocarcinogênese diferente em pacientes geriátricos.

Para o tratamento do $\mathrm{CHC}$ há as modalidades de tratamento não cirúrgicas, geralmente paliativas, como a radiofrequência, alcoolização e quimioembolização. Somente o transplante e a ressecção hepática possuem o intento curativo. A cirúrgica é restrita a pacientes com ou sem cirrose ou com cirrose com classificação ChildPugh A sem hipertensão portal. Nos países ocidentais esse contingente representa menos de $5 \%$ dos pacientes, com alta taxa de recorrência pós-ressecção9.

Diversos estudos analisaram o desfecho da ressecção hepática em pacientes jovens e idosos. Mesmo com os pacientes idosos possuindo mais comorbidades, não se constatou impacto nas taxas de complicações pós-operatórias na análise geral dos estudos. Os dados de mortalidade ainda variam na literatura, apresentando maiores taxas de complicações em estudos mais antigos ${ }^{25}$ e menores em estudos mais recentes, resultado da melhor qualidade técnica e aperfeiçoamento por parte dos cirurgiões9. Diversos estudos consideram a ressecção do $\mathrm{CHC}$ segura em pacientes idosos, contanto que as comorbidades sejam controladas $7,9,11,17,19,20,24,26$.

Mesmo com expectativa de vida esperada menor, em pacientes idosos selecionados a sobrevida de cinco 
anos pós-ressecção do $\mathrm{CHC}$ foi de 28 a $58 \%$, equivalente à de pacientes jovens. Esses dados justificam ressecção hepática extensa em pacientes dessa faixa etária com $\mathrm{CHC} \mathrm{C}^{9,11,17,19,20,24,26 \text {. }}$

\section{Metástases colorretais}

Aproximadamente 437 mil pessoas morrem anualmente por câncer colorretal no mundo, colocando-o como a terceira causa de morte por câncer. Cerca de $80 \%$ dos tumores colorretais são diagnosticados em pacientes entre 65 e 85 anos 5,9, porém é importante observar-se que, mesmo com a prevalência da neoplasia colorretal aumentando em pacientes idosos, a sobrevida nessa faixa etária não cresceu o que se explica pelo fato de que os idosos são menos submetidos ao tratamento cirúrgico curativo do tumor primário e suas metástases ${ }^{23}$.

O fígado é o órgão mais frequentemente atingido por metástases à distância da neoplasia colorretal. As sincrônicas estão presentes em cerca de $20 \%$ dos com câncer colorretal e metacrônicas em aproximadamente 20-50\% ${ }^{8,12}$. Sabe-se que a ressecção hepática é a única opção terapêutica de cura para pacientes com metástases apenas no fígado9,10,17. Entretanto, os estudos demonstraram que a taxa de ressecção de metástases hepáticas de tumor colorretal é de 8 a $20 \%$ em pacientes com mais idade ${ }^{8,10}$. Essa justificativa tornase válida, pois quando se contrapõem os benefícios de ressecção cirúrgica de metástases hepáticas contra os potenciais riscos da cirurgia, muitos médicos ainda ficam relutantes em indicar o tratamento cirúrgico em idosos. Embora essa abordagem não se baseie em evidências científicas, é provável que as preocupações com a morbidade e a mortalidade pós-operatórias em idosos possam influenciar a decisão de não oferecer a operação a eles ${ }^{8}$.

Recentes melhoras nas técnicas de ressecção hepática e nas intervenções anestésicas melhoraram muito os resultados. A mortalidade pós-operatória caiu nas últimas décadas e, nos dias atuais, o cuidado per-operatório culminou em mortalidade variável entre 0 e $11 \%$ para procedimentos de ressecção de cólon e de metástase hepática combinados. Ainda, a quimioterapia neoadjuvante é utilizada com a intenção de melhorar a sobrevida livre de doença pós-ressecção hepática, e, também, proporcionar aos pacientes com doença inicial irressecável operação com potencial curativo ${ }^{8}$.

A ressecção de metástases hepáticas de câncer colorretal em pacientes idosos tem-se mostrado segura, com morbimortalidade semelhantes na população não idosa e idosa ${ }^{1,24,5,6,6,7,10,13,14,15,16,27}$.

A sobrevida de cinco anos após a ressecção hepática relatada por diversos estudos variou de 28 a 58\%, com sobrevida média de 16 a 46 meses, semelhante aos pacientes jovens. Diversos estudos recentes revelaram que a idade por si não é fator de risco para o desfecho. Os achados indicam que os idosos com metástase hepática de neoplasia colorretal se beneficiam no mesmo grau que os pacientes jovens. Ainda mais, a ressecção hepática para metástases de tumor colorretal deveria ser a opção preferida em idosos com comorbidade controlada, pelos resultados encorajadores encontrados ${ }^{1,4,5,6,7,10,13,14,16,27}$.

\section{Tumores do hilo biliar}

O tumor do hilo biliar (tumor de Klatskin) representa a forma mais comum de neoplasia da via biliar. O pico de incidência da doença ocorre na sétima década de vida ${ }^{17}$. A sua história natural é reservada, com poucos relatos de sobreviventes após cinco anos do diagnóstico. Embora o tratamento cirúrgico seja difícil e inquestionável, ele é limitado a centros especializados onde a ressecção radical é a única chance potencial de cura.

Devido à proporção cada vez maior de pacientes em idade avançada diagnosticados com esse tumor, são importantes os questionamentos sobre a extensão da ressecção que pode ser realizada com segurança e se esse tratamento aumentaria a sobrevida. Infelizmente não há estudos adequados que avaliem essas questões em relação à idade. Há apenas duas publicações na literatura que sugerem que a sobrevida em cinco anos, bem como sobrevida média, é semelhante no grupo de pacientes jovens e idosos. Apesar da ausência de outros estudos comparativos de idade, os dados de sobrevida desses dois pequenos estudos demonstram que os pacientes idosos podem se beneficiar de ressecção hepática extensa assim como os jovens, desde que bem selecionados.

\section{Outras indicações de hepatectomia}

$\mathrm{Na}$ literatura há, também, séries que comparam a ressecção de metástases de outros tumores não colorretais e lesões benignas como cistos hepáticos, hemangiomas, hiperplasia nodular focal, adenoma hepatocelular e hepatolitíase entre pacientes jovens e idosos. Foi averiguado que o desfecho após o procedimento no que se refere à morbidade, mortalidade e sobrevida é semelhante em idosos em comparação com jovens ${ }^{6,15,18}$. No entanto, esses dados são inseridos em trabalhos maiores onde a frequência do carcinoma hepatocelular, tumores do hilo biliar e metástases de neoplasias colorretais destacam-se, não sendo possível comparar separadamente os resultados da cirurgia nessas doenças em idosos.

\section{Segurança da hepatectomia}

Observou-se que com as hepatectomias foi obtida redução da mortalidade nos últimos anos. Em 1970, ela era de $20 \%$; atualmente é menor que $5 \%^{3}$.

Ao mesmo tempo, houve avanços nos cuidados perioperatórios. Ocorreu melhora na seleção e no preparo pré-operatório dos pacientes, avanços das técnicas anestésicas e de cuidados intensivos pósoperatórios. Houve melhor compreensão da anatomia 
segmentar hepática e de inovações em dispositivos de transecção do tecido hepático, definindo técnica cirúrgica mais refinada com controle mais acurado da hemostasia. Estes fatos, melhoraram a segurança das operações. Inovações permitiram a ampliação e a modificação constante dos critérios de elegibilidade para hepatectomias incluindo pacientes com doença hepática crônica associada, principalmente os idosos.

Diversos estudos demonstraram que as ressecções hepáticas estão sendo cada vez mais realizadas em pacientes idosos, apresentando resultados encorajadores. No entanto, estudos comparativos que especificamente analisam o papel desempenhado pela idade sobre o resultado da ressecção hepática em indivíduos jovens e idosos são ainda pouco frequentes na literatura frente ao crescente número de procedimentos realizados em idosos ${ }^{17}$. Estudos prévios realizados na Europa, América do Norte e Ásia demonstraram que há segurança em hepatectomias em idosos com taxas de morbidade e mortalidade que variam entre 9 e $52,5 \%$ e 0 a $11 \%$, respectivamente.

\section{CONCLUSÃO}

A idade não influencia a morbidade e mortalidade intra-hospitalar, bem como a sobrevida de pacientes submetidos à hepatectomia, desde que sejam bem selecionados. Deve-se encorajar esse procedimento em pacientes de mais idade, face ao aumento do número de indivíduos candidatos ao tratamento cirúrgico de suas doenças hepáticas.

\section{REFERÊNCIAS}

1. Adam R, Frilling A, Elias D, et al. and the LiverMetSurvey Centres. Liver resection of colorectal metastases in elderly patients. British Journal of Surgery 2010; 97: 366-376

2. Aguiar, LRF, Nassif PAN, Ribas CAPM, Czeczko NG, Ribas MM, Marinho-Júnio $\mathrm{CH}$,Wendler E. Regeneração do fígado após hepatectomia parcial em ratos submetidos à hipertensão portal pós-hepática. ABCD Arq Bras Cir Dig. 24(2):144-151, 2011.

3. Aldrighetti $L$, Arru $M$, Caterini $R$, et al. Impact of advanced age on the outcome of liver resection. World J Surg 2003;27:1149- 1154

4. Bockhorn M, Sotiropoulos GC, Sgourakis G, et al. Major liver resections in the elderly: is an aggressive approach justified? Int J Colorectal Dis 2009;24:83-86

5. Brand MI, Saclarides Tj, Dobson HD. Liver resection for colorectal cancer: liver metastases in the aged. Am Surg 2000;66:412

6. Brunken C, Rogiers X, Malago M. Is resection of colorectal liver metastases still justified in very elderly patients. Chirurg 1998;69:1334
7. Caratozzolo $E$, Massani $M$, Recordare $A$, et al. Liver resection in elderly: comparative study between younger and older than 70 years patients. Outcomes and implications for therapy. G Chir 2007:28:419-424

8. Carino NL. Liver resection for colorectal liver metastases in older patients. Critical Review in Oncology/Hematology 2008;67:273278

9. Ferrero A, Vigano L, Polastri R. Hepatectomy as treatment of choice for hepatocellular carcinoma in elderly cirrhotic patients. World J Surg 2005;29:1101- 1105

10. Fong Y. Fong Y, Blumgart LH, Fortner JG. Pancreatic or liver resection for malignancy is safe and effective for the elderly. Ann Surg 1995;222:426

11. Hanazaki K, Kajikawa S, Shimozawa N. Hepatic resection for hepatocellular carcinoma in the elderly. J Am Coll Surg 2001;192:38

12. Instituto Nacional do Câncer. Site do INCA. Rio de Janeiro: INCA; 2011

13. Jansen PLM. Liver disease in the elderly. Best Practice and Research Clinical Gastroenterology, 2002;16:149-158

14. Mann CD, Neal CP, Pattenden CJ, et al. Major resection of hepatic colorectal liver metastases in elderly patients e an aggressive approach is justified. EJSO 2008;34:428-432

15. Menon KV, Mukhtar AA, Aldouri A, et al. Outcomes after major hepatectomy in elderly patients. J Am Coll Surg 2006;203:677-683

16. Papamichael D, Audisio R, Horiot JC, et al. Treatment of the elderly colorectal cancer patient: SIOG expert recommendations. Annals of Oncology 2009;20:5-16

17. Petrowsky $\mathrm{H}$, Clavien PA. Should we deny surgery for malignant hepato-biliary tumors to elderly patients? World J Surg 2005;29:1093-1100

18. Pisani, P. Estimates of the worldwide mortality from 25 cancers in 1990. Int J Cancer 1999;83:18-29

19. Poon RT, Fan ST, Lo CM. Hepatocellular carcinoma in the elderly: results of surgical and nonsurgical management. Am J Gastroenterol 1999;94:2460

20. Portolani N, Baiocchi GL, Coniglio A, et al. Limited liver resection: a good indication for the treatment of hepatocellular carcinoma in elderly patients. Jpn J Clin Oncol 2011;41:1358-65

21. Quaglia A, Capocaccia R, Micheli A, et al and the EUROCARE-3 Working Group. A wide difference in cancer survival between middle aged and elderly patients in Europe. Int J Cancer 2007;120:2196-2201

22. SEER Cancer Statistics Review 1975-2001, 2011.

23. Temple LK, Hsieh L, Wong WD. Use of surgery among elderly patients with stage IV colorectal cancer. J Clin Oncol 2004;22(17):3475-84

24. Wu CC, Chen JT, Ho WL et al. Liver resection for hepatocellular carcinoma in octogenarians. Surgery 1999;125:332

25. Yamamoto K, Takenaka K, Matsumata T, et al. Right hepatic lobectomy in elderly patients with hepatocellular carcinoma. Hepatogastroenterology 1997;44:514

26. Yeh CN, Lee WC, Jeng LB. Hepatic resection for hepatocellular carcinoma in elderly patients. Hepatogastroenterology 2004;51:219

27.Zieren HU, Muller JM, Zieren J. Resection of colorectal liver metastases in old patients. Hepatogastroenterology 1994;41:34 